\title{
Uma Proposta Orientada a Perfis de Capacidade de Processo para Evolução da Melhoria de Processo de Software (Tese de Doutorado - UNICAMP)
}

\author{
Clênio F. Salviano ${ }^{1,2}$ e Mario Jino ${ }^{2}$ (Orientador) \\ ${ }^{1}$ Divisão de Melhoria de Processo de Software \\ Centro de Pesquisas Renato Archer \\ Ministério da Ciência e Tecnologia (DMPS CenPRA MCT), \\ Rodovia Dom Pedro I, km 143,6, CEP 13069-901, Campinas - SP - Brasil \\ ${ }^{2}$ Departamento de Engenharia de Computação e Automação Industrial \\ Faculdade de Engenharia Elétrica e de Computação \\ Universidade Estadual de Campinas (DCA FEEC UNICAMP) \\ Cidade Universitária “Zeferino Vaz”, CEP 13081-970, Campinas, SP - Brasil \\ clenio.salviano@cenpra.gov.br,jino@dca.fee.unicamp.br
}

\begin{abstract}
Software process improvement based on the staged architecture's fixed maturity levels of SW-CMM and CMMI models do not support the diversity of software intensive organizations context and strategic objectives. This research evolves the current process improvement area taking advantage of the flexibility of ISO/IEC 15504 continuous architecture towards a better alignment between an improvement with each organization's specific context and strategic objectives, especially of micro and small ones. Three generations of architectures for process capability models (fixed staged, closed continuous, open continuous) are identified. A process capability profile driven, software and any other knowledge intensive human work, process engineering is proposed. An exemplar approach for this engineering is developed, with a set of properties, a unified model, an improvement cycle and a measurement set, as well as a method for establishing process capability profile. These proposals are validated by analyses, practical applications and a research agenda.
\end{abstract}

Resumo. Melhoria de processo de software baseada nos níveis de maturidade fixos da arquitetura estagiada dos modelos SW-CMM e CMMI não atendem à diversidade de contextos e objetivos estratégicos das organizações intensivas em software. Esta pesquisa evoluí a área de melhoria de processo com a utilização da flexibilidade da arquitetura contínua da ISO/IEC 15504 para um melhor alinhamento da melhoria com o contexto e objetivos estratégicos específicos de cada organização, principalmente as micro e pequenas. São identificadas três gerações de arquiteturas de modelos de capacidade de processo (estagiada fixa, contínua fechada e contínua aberta). É proposta uma engenharia de processo, de software e de qualquer outro trabalho humano intensivo em conhecimento, dirigida por perfis de capacidade de processo. $E$ desenvolvida uma abordagem exemplo dessa engenharia com um conjunto de propriedades, um 
modelo unificado, um ciclo de melhoria e um conjunto de medições, bem como um método para o estabelecimento de perfis de capacidade de processo. As propostas são validadas com análises, aplicações práticas e uma agenda de pesquisa.

\section{Introdução}

Esse artigo apresenta um resumo da uma tese de doutorado defendida e aprovada em março de 2006 na Faculdade de Engenharia Elétrica e de Computação da Universidade Estadual de Campinas (FEEC-UNICAMP) [Salviano 2006]. A Seção 2 desse artigo apresenta a área de pesquisa. A Seção 3 apresenta problemas e oportunidades considerados na pesquisa. A Seção 4 apresenta objetivos e propostas da pesquisa. A Seção 5 apresenta a metodologia e o processo da pesquisa. As Seções 6, 7, 8 e 9 apresentam respectivamente as quatro propostas principais da pesquisa: gerações de arquitetura de modelos de capacidade, engenharia de processo, abordagem PRO2PI para engenharia de processo e um método para estabelecimento de PRO2PI. A Seção 10 apresenta experimentações e análises. A Seção 11 apresenta as principais contribuições da pesquisa e a Seção 12 apresenta a conclusão.

\section{Apresentação}

O termo 'engenharia de software' foi definido em uma conferência da NATO (North Atlantic Treat Organization) em 1968, "como uma provocação, para implicar na necessidade da construção de software ser baseada nos moldes dos fundamentos teóricos e disciplinas práticas, que são tradicionais nos tipos de engenharia já estabelecidos" [Naur e Randell 1969, p. 8]. Desde então a comunidade tem procurado entender a natureza das atividades de software e sua relação com as outras disciplinas da engenharia, e desenvolver métodos, técnicas, modelos e ferramentas para a engenharia de software. Tem havido um amadurecimento e avanço na área, porém software é ainda mais "produzido" e não exatamente "engenhariado" [Pollice 2005]. Segundo Herbsleb et al. [1997] "enquanto Fred Brooks nos avisava que não haveria uma única solução 'bala de prata' (silver bullet) para as dificuldades essenciais do desenvolvimento de software [Brooks 1986], Watts Humphrey e outros no Software Engineering Institute (SEI) estavam ocupados colocando idéias que viriam a ser o modelo SW-CMM" [Hersberg et al. 1997, p. 31].

A versão 1.1 do modelo SW-CMM (Capability Maturity Model for Software) [Paulk et al. 1994], lançada em 1993, estabeleceu a área de melhoria de processo de software, como uma alternativa às abordagens tradicionais da engenharia de software. A área foi estabelecida com sucesso principalmente na comunidade da prática com o objetivo de orientar a melhoria do processo de projetos de desenvolvimento de software por encomenda, como meio para a melhoria das organizações intensivas em software, tendo como referência níveis fixos de maturidade. O modelo SW-CMM define a arquitetura estagiada com cinco níveis de maturidade fixos.

Como uma alternativa à rigidez da arquitetura estagiada, foi proposta a arquitetura contínua pela ISO/IEC 15504, que também é conhecida como modelo SPICE (Software Process Improvement and Capability dEtermination). A ISO/IEC 15504 propõe modelos com duas dimensões: um conjunto de áreas de processo e uma 
sequiência de níveis de capacidade de processo. Com a arquitetura contínua, cada organização pode escolher um perfil de capacidade de processo, composto por áreas de processo selecionadas, cada uma em um determinado nível de capacidade, para orientar a melhoria. A ISO/IEC 15504 já teve três versões, em 1995, 1998 e 2003, com o aumento da flexibilidade a cada versão. A ISO/IEC 15504-2 [2003] define um framework para modelos de capacidade de processo e processos para avaliação de processo e o modelo exemplo de capacidade de processo ISO/IEC 15504-5 [2006] para a engenharia de software.

O SW-CMM foi evoluído para um framework de modelos integrados (CMMI: Capability Maturity Model Integration) [Chrissis et al. 2003] alinhado com a ISO/IEC 15504. Os modelos do CMMI são disponibilizados tanto na representação estagiada, como o SW-CMM, como na representação contínua, como a ISO/IEC 15504-5. Outros modelos de capacidade de processo relevantes são o FAA iCMM [Ibrahim 2000], ITsqc eSCM-SP, PMI OPM3 e MPS.BR MR-MPS [Weber et al. 2005].

Uma parte da comunidade tem defendido mais a arquitetura estagiada, como, por exemplo, Curtis [1998] e Weber et al. [2005]; outra tem defendido mais a arquitetura contínua, como, por exemplo, Ibrahim [2000] e Shaeffer [2004]; e outra tem buscado diferentes formas de composição entre as duas arquiteturas, como, por exemplo, Olson [2003] e Kasse [2004]. O cenário atual é composto pelo estabelecimento com sucesso da melhoria baseada nos modelos estagiados (SW-CMM e CMMI) e o reconhecimento das limitações naturais dessa abordagem, como, por exemplo, Conradi e Fuggetta [2002] e Rifkin [2002]. A arquitetura contínua ainda não está estabelecida no estado da prática, sendo uma potencialidade para evoluir as abordagens correntes de melhoria de processo.

Apesar de ser uma força no estado da prática do setor de software, a área de melhoria de processo ainda não é um tópico popular de pesquisas mais rigorosas, principalmente nas universidades [Card 2004]. Como a área tem sido impulsionada pela prática, os diferentes modelos e abordagens são considerados concorrentes, embora compartilhem os mesmos princípios básicos. A insuficiência de pesquisas nessa área, tem limitado a identificação desses princípios básicos, que é papel da ciência.

\section{Problemas e oportunidades}

Esta pesquisa identifica que o não entendimento dos princípios básicos e a falta de apoio metodológico para utilização da flexibilidade oferecida pelos modelos com arquitetura contínua são os principais impedimentos técnicos para a evolução da melhoria de processo com o uso mais disseminado dos modelos contínuos. Identifica também que as principais limitações da atual melhoria de processo de software podem ser superadas com uma combinação adequada das arquiteturas contínua e estagiada.

Do ponto de vista do setor de software existe a demanda por uma melhor efetividade dos programas de melhoria de processo e de um melhor alinhamento ao contexto e objetivos estratégicos de cada organização, principalmente as micro e pequenas. Como os modelos com arquitetura estagiada definem níveis de maturidade fixos, para projetos de desenvolvimento de software sob encomenda, esses níveis podem não ser viáveis, relevantes e suficientes, ou mesmo não aplicáveis, a algumas organizações como, por exemplo, micro e pequenas organizações orientadas à evolução 
e comercialização de produtos. Também tem aumentado a quantidade de organizações que utilizam elementos de mais de um modelo como referência para a melhoria como, por exemplo, os modelos do CMMI, ISO 9001: 2000 e PMBOK Third Edition.

\section{Objetivos e propostas da pesquisa}

Esta pesquisa foi iniciada com o objetivo de desenvolver apoio metodológico para a escolha de processos do modelo ISO/IEC TR 15504-5 para orientar a melhoria de uma organização intensiva em software, alinhada ao contexto e objetivos estratégicos dessa organização. Com o desenvolvimento e utilização desse apoio metodológico e com a evolução da área esse objetivo foi ampliado. O objetivo principal da pesquisa passou a ser explorar as possibilidades de flexibilidade oferecidas pela arquitetura contínua preservando as vantagens da arquitetura estagiada. Esse objetivo principal foi desdobrado em cinco objetivos relacionados.

O objetivo 1 é entender melhor as semelhanças, diferenças e relacionamentos das arquiteturas estagiada e contínua de modelos de capacidade de processo e identificar os princípios básicos unificadores das arquiteturas. O objetivo 2 é evoluir a abordagem corrente de melhoria de processo de software, com o desenvolvimento de apoio metodológico para a utilização da flexibilidade dos modelos contínuos para a melhoria de processo orientada ao contexto e objetivos estratégicos de uma organização. O objetivo 3 é desenvolver uma abordagem para essa evolução da melhoria de processo. O objetivo 4 é experimentar essa nova abordagem principalmente para início de um programa de melhoria em micro e pequenas organizações intensivas em software. O objetivo 5 é estabelecer uma agenda para continuação das pesquisas.

\section{Metodologia e processo da pesquisa}

O processo dessa pesquisa foi orientado por uma metodologia baseada na abordagem 'indústria-como-laboratório' [Potts 1993] com em três fases (desenvolvimento, caracterização e formalização) cada uma com ciclos de exploração, aplicação e consolidação.

Para pesquisas em engenharia de software, Potts defende a abordagem 'indústria-como-laboratório' (industry-as-laboratory) como uma abordagem complementar á tradicional 'pesquisa-depois-transfere' (research-then-transfer). Na abordagem indústria-como-laboratório, as idéias para pesquisa são baseadas em problemas práticos, e são refinadas em estudos de casos contínuos e incrementais. Com isto, segundo Potts, "os domínios de pesquisa e aplicação tendem a convergirem, e a transferência de tecnologia e o processo de avaliação são realizados nos estágios iniciais do programa de pesquisa" [Potts 1993, p. 22].

O processo desta pesquisa foi realizado em três fases: desenvolvimento, caracterização e formalização. A primeira fase, chamada de desenvolvimento, foi realizada de novembro de 1998 a outubro de 2002 e focou no desenvolvimento e aplicação de uma Abordagem para Melhoria de Processo (AMP1), baseada no ciclo de melhoria da ISO/IEC 15504 e com a utilização do modelo contínuo da ISO/IEC TR 15504-5 [1998]. Para essa abordagem foi desenvolvido também um Método para Escolha de Processos (MEP1) para orientar uma melhoria alinhada ao contexto e objetivos estratégicos da organização. A segunda fase, denominada de caracterização, foi realizada de setembro de 2003 a outubro de 2004. O principal marco dessa fase foi o 
desenvolvimento do artigo com a definição das gerações de arquitetura de modelos e a primeira versão de PRO2PI. A terceira fase, denominada de formalização, foi realizada de novembro de 2004 a outubro de 2005. Nessa fase, PRO2PI foi consolidado, como um exemplo de abordagem para uma engenharia de processo dirigida por perfis de capacidade de processo. Para apoiar a utilização de PRO2PI foi também desenvolvido e utilizado um método para o estabelecimento de PRO2PI.

\section{Proposta 1: Gerações de arquitetura de modelos de capacidade}

O conceito de Perfil de Capacidade de Processo (PCP) é o conceito unificador entre as arquiteturas estagiada e continua. $\mathrm{O}$ conceito de $\mathrm{PCP}$ e dois outros conceitos auxiliares, que são área de processo e nível de capacidade de processo, formam a base para esta primeira proposta de três gerações de arquitetura de modelos de capacidade de processo, que substitui a caracterização corrente de estagiada versus contínua. A Figura 1 relaciona as três gerações propostas, que são denominadas de estagiada fixa, contínua fechada e contínua aberta, e identifica suas principais características.

\begin{tabular}{|c|c|c|c|}
\hline & Primeira geração & Segunda geração & Terceira geração \\
\hline $\begin{array}{c}\text { Framework ou } \\
\text { modelo principal }\end{array}$ & $\begin{array}{c}\text { Modelo } \\
\text { SW-CMM v. 1.1 }\end{array}$ & $\begin{array}{c}\text { Framework } \\
\text { ISO/IEC TR 15504 }\end{array}$ & $\begin{array}{c}\text { Framework } \\
\text { ISO/IEC 15504 }\end{array}$ \\
\hline Ano de lançamento & 1993 & 1998 & 2003 \\
\hline Nome atual & Estagiado & Contínuo & Contínuo \\
\hline Outros modelos & $\begin{array}{c}\text { CMMI-SW Est. v1.1 } \\
\text { MR-MPS }\end{array}$ & $\begin{array}{c}\text { ISO/IEC 15504-5 } \\
\text { iCMM,CMMI-SW Con }\end{array}$ & $\begin{array}{c}\text { Utilizações de } \\
\text { PRO2PI }\end{array}$ \\
\hline Nome proposto & Estagiado fixo & Contínuo fechado & Contínuo aberto \\
\hline $\begin{array}{c}\text { Principais } \\
\text { elementos fixos } \\
\text { (estabilidade) }\end{array}$ & $\begin{array}{c}\text { Hierarquia de } \\
\text { de Processo (PCPs) }\end{array}$ & $\begin{array}{c}\text { Níveis de capacidade, } \\
\text { Áreas de processo (AP) }\end{array}$ & $\begin{array}{c}\text { Níveis de capacidade, } \\
\text { e requisitos para } \\
\text { APs e PCPs }\end{array}$ \\
\hline $\begin{array}{c}\text { Principais } \\
\text { elementos variáveis PCPs } \\
\text { (flexibilidade) }\end{array}$ & $\begin{array}{c}\text { Interpretação } \\
\text { dos PCPs }\end{array}$ & $\begin{array}{c}\text { Escolha e interpretação } \\
\text { de PCPs }\end{array}$ & $\begin{array}{c}\text { Escolha e definição } \\
\text { de APs, e escolha e } \\
\text { interpretação de PCPs }\end{array}$ \\
\hline Abordagens & MPS, IDEAL & MEP1, Toolkit SEI & PRO2PI \\
\hline $\begin{array}{c}\text { Comentários } \\
\text { e implicações } \\
\text { para a melhoria }\end{array}$ & $\begin{array}{c}\text { Sucesso, fundamental } \\
\text { para estabelecer a } \\
\text { área de MPS }\end{array}$ & $\begin{array}{c}\text { Evolução, separação } \\
\text { entre essência (APs e } \\
\text { NCs) e uso (PCPs) }\end{array}$ & $\begin{array}{c}\text { Evolução, essência (NCs) } \\
\text { e uso (APs e PCPs), } \\
\text { nova área: PCDE }\end{array}$ \\
\hline
\end{tabular}

Figura 1 - Gerações de modelos de capacidade de processo

\section{Proposta 2: Engenharia de Processo}

Um perfil de capacidade de processo é um modelo que representa um processo segundo $o$ aspecto de capacidade de processo. O processo de uma organização deve ser representado por um perfil de capacidade de processo, que é uma abstração do processo, segundo o aspecto capacidade de processo. O par consistente formado por perfil de capacidade de processo e processo, é ilustrado na Figura 2.

Nesse caso tudo que está representado no perfil existe no processo e no processo existe apenas aquilo que está representado no perfil. Desta forma o princípio da 'substitucionalidade' limitada [Bézivin 2003] se aplica. Quando o perfil de capacidade de processo representa o processo atual de uma organização ele é um modelo descritivo. 
Por outro lado, quando o perfil de capacidade de processo representa o processo alvo de uma melhoria em uma organização ele é um modelo prescritivo.

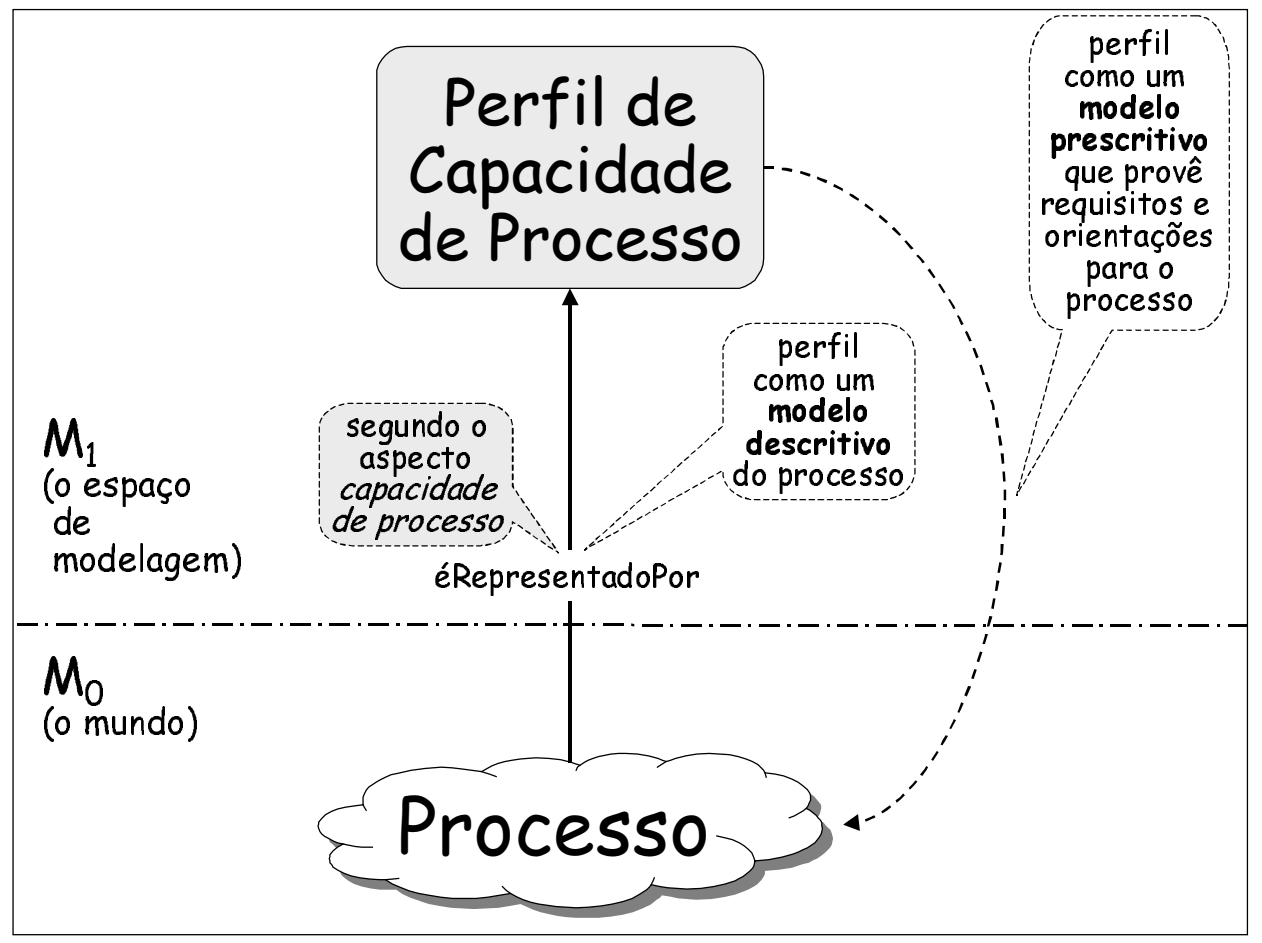

Figura 2 - Par consistente perfil de capacidade de processo e processo

Outra forma de entender a relação entre o perfil e o processo é com o seguinte questionamento: se um nível de maturidade do modelo CMMI-SE/SW ou do modelo MPS-BR MR-MPS tivesse sido definido uma determinada organização, em um determinado momento, qual seria o nível de maturidade de forma a representar o processo atual ou o processo alvo para uma melhoria alinhada ao contexto e objetivos estratégicos da organização. Desta forma a melhoria de processo deve ser evoluída para uma engenharia que trate esse par consistente no centro dessa engenharia. A segunda proposta é então uma Engenharia de Processo de Software Dirigida por Perfil de Capacidade de Processo (Process Capability Profile Driven Process Engineering PCDE) como uma evolução da atual melhoria de processo de software baseada em modelos de maturidade,

Seguindo a tendência de expansão da abrangência da melhoria de processo de software para melhoria de processo de software e de sistemas, evidenciada por exemplo pelo CMMI e ISO/IEC 15504, a engenharia de processo de software também deve ter essa expansão. Para isso é proposta a expressão "de software e de qualquer outro trabalho humano intensivo em conhecimento" na qual é ressaltada a abrangência dessa engenharia para software, que tem sido a área na qual é mais utilizada, e caracterizado de forma mais precisa a abrangência maior da engenharia, que é qualquer trabalho humano intensivo em conhecimento, incluindo trabalho de software. A escolha dessa terminologia é baseada no termo "trabalhador do conhecimento" (knowledge worker) [Drucker 1992].

Esta segunda proposta então tem a seguinte definição mais completa: 
\{(Engenharia de processo) (de Software e de qualquer outro Trabalho Humano Intensivo em Conhecimento) Dirigida por (Perfis de Capacidade de Processo)\}

ou de forma mais curta

\title{
\{(Engenharia de processo) Dirigida por (Perfis de Capacidade de Processo) $\}$
}

\author{
é definida como:
}

(1) a aplicação de engenharia à definição, utilização, manutenção, estabelecimento, medição, mudança, melhoria e co-evolução de pares consistentes de perfil de capacidade de processo e processo para uma melhoria de processo dirigida por perfis de capacidade de processo, em processos de software e de qualquer outro trabalho humano intensivo em conhecimento, como meio para atingir excelência organizacional alinhada ao contexto e objetivos estratégicos;

(2) a aplicação de engenharia ao desenvolvimento e manutenção de modelos de capacidade de processo para contextos ou domínios estratégicos mais específicos; e

(3) o estudo das abordagens como em (1) e (2).

Esta definição é baseada em uma combinação de definições de engenharia [Shaw 1990], engenharia de software [Abran et al. 2004], engenharia de processo de software [El Emam 2004], melhoria de processo de software [Zahran 1998] e engenharia dirigida por modelos [Bézivin 2003].

\section{Proposta 3: Abordagem PRO2PI para engenharia de processo}

A terceira proposta é uma abordagem exemplo para a engenharia de processo PCDE que oriente a experimentação, validação e evolução de PCDE. A abordagem é denominada de abordagem PRO2PI (Perfil de Capacidade de Processo para Melhoria de Processo, em inglês, Process Capability Profile to Process Improvement). PRO2PI é uma abordagem para o desenvolvimento de modelos mais específicos e para uma melhoria de processo dirigida por perfis de capacidade de processo. O termo PRO2PI é também utilizado para designar o perfil de capacidade de processo definido e utilizado para dirigir a melhoria de processo. A Figura 3 ilustra uma visão geral da utilização de PRO2PI.

A partir do contexto de negócio de um segmento, como, por exemplo, o segmento bancário, ou de um domínio, como, por exemplo, engenharia de requisitos, pode ser definido um modelo mais específico. Esse modelo pode ser composto por áreas de processo ou perfis de capacidade de processo mais específicos para um segmento ou domínio. Esse modelo mais específico pode conter boas práticas selecionadas dos modelos de capacidade de processo, modelos de outros tipos ou de qualquer outra fonte de forma a representar orientações relevantes no segmento ou domínio. Esse uso é representado pela função "defineM" (define modelo) da Figura 3.

Um modelo mais específico pode ser de qualquer uma das três gerações de arquiteturas. Caso seja um modelo estagiado fixo, ele será composto por uma hierarquia de perfis de capacidade de processo, como, por exemplo, a hierarquia do modelo $P M M M$ para o domínio de gerência de projeto e do modelo $K M M M$ para o domínio de gerência de conhecimento. Caso seja um modelo contínuo fechado, ele será composto por um conjunto de áreas de processo, como, por exemplo, as áreas de processo do 
modelo Automotive SPICE para o segmento de fornecedores de software para automóveis e as do modelo OOSPICE para o domínio de desenvolvimento de software orientado a componentes. Esses modelos podem ter sugestões de hierarquia de perfis de capacidade de processo. Caso seja um modelo contínuo aberto, ele será composto de especializações dos níveis de capacidade de processo e sugestões para as áreas de processo.

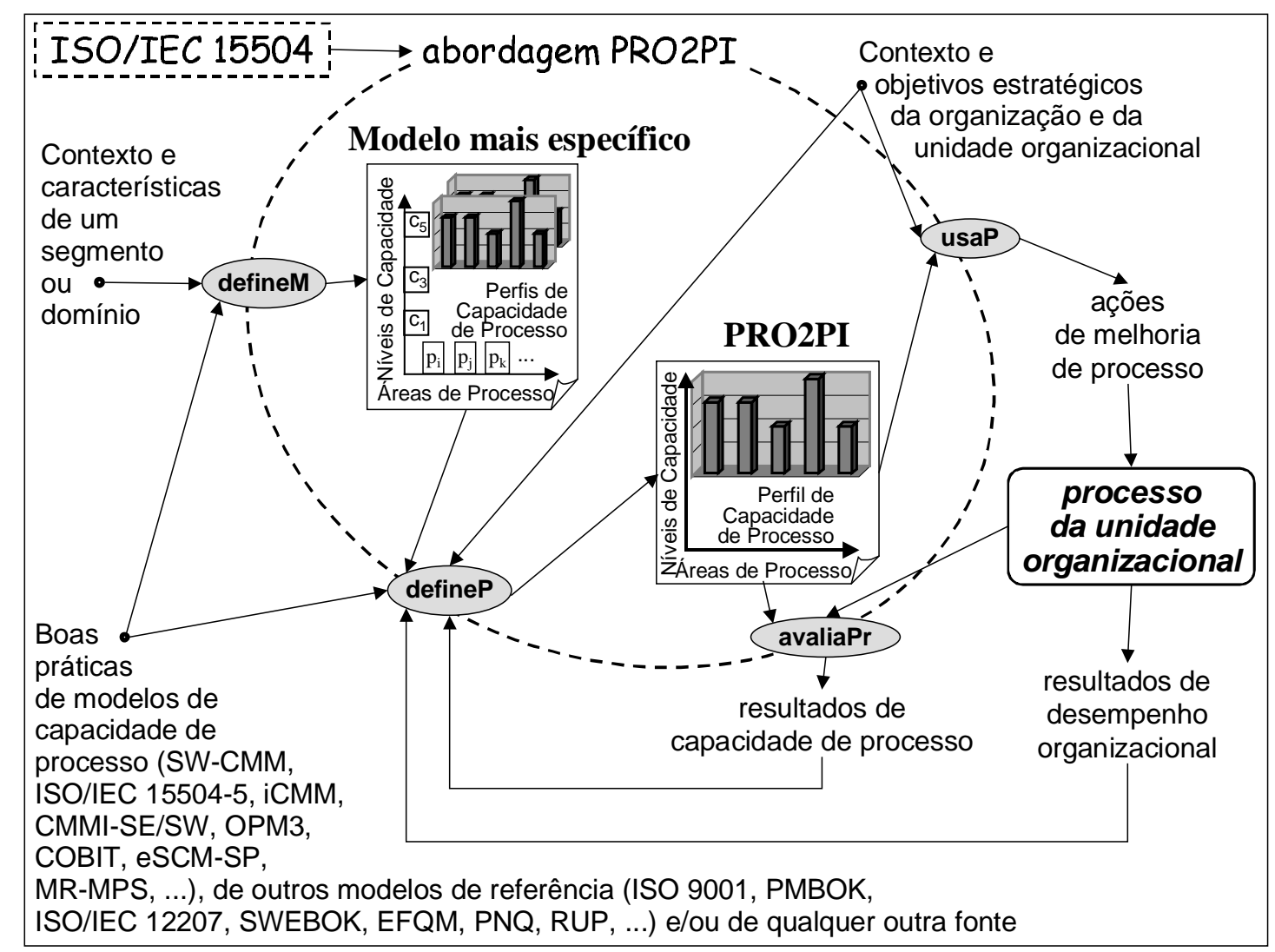

Figura 3- visão geral da utilização de PRO2PI.

Uma organização seleciona elementos de um ou mais modelos de referências, mais genéricos ou mais específicos de um segmento ou domínio, e define um perfil de capacidade de processo que representa os elementos selecionados e outros elementos apropriados. Um ciclo de melhoria de processo é realizado buscando evoluir os processos para atender a esse perfil. Esse perfil é alinhado com o contexto e objetivos estratégicos da organização. O perfil pode conter boas práticas de referência selecionadas dos modelos mais genéricos existentes e de outras fontes, de forma a representar orientações relevantes à organização. Esse perfil pode ser alterado a qualquer momento em função de novas percepções, alterações do contexto ou dos objetivos estratégicos, e dos resultados da utilização da versão corrente do perfil. Esse uso é representado pela função "defineP" (define ou atualiza perfil) da Figura 3. O uso desse perfil para orientar as ações de melhoria é representado pela função "usaP" (usa perfil) da Figura 3. Neste caso as ações de melhoria devem ser suficientes para que o processo resultante atenda a todos os requisitos representados no perfil e apenas a estes requisitos. 
O processo da unidade organizacional pode examinado com uma avaliação de capacidade de processo em relação ao perfil de capacidade de processo. Esse exame é representado na Figura 3 pela função "avaliaPr" (avalia capacidade de processo em relação a um PRO2PI). Os resultados de capacidade de processo gerados por essa avaliação podem ser utilizados como referências adicionais para a definição, ou alteração, do perfil de capacidade de processo.

A abordagem PRO2PI pode ser descrita por meio de quatro componentes. O primeiro componente é um conjunto de oito propriedades (relevante, oportuno, viável, sistêmico, representativo, rastreável, específico e dinâmico) que um PCP deve ter, em um grau suficiente, para ser um PRO2PI;

O segundo componente é um modelo que (i) unifica os elementos das estruturas dos modelos de capacidade de processo, especialmente dos modelos iCMM v2.0, CMMI-SE/SW v1.1, ISO/IEC 15504-5 e MR-MPS v1.0, e com isto permite a representação de qualquer elementos desses modelos em um PRO2PI, (ii) permite a representação em um PRO2PI de elementos selecionados de outros modelos de referência, especialmente PMBOK e ISO 9001, (iii) é representado com um diagrama de classes e pode ser representado como um metamodelo, e (iv) sintetiza todos os elementos desses modelos em uma única classe $\mathrm{Cl}$ _ReferencePractice (prática de referência), que é especializada inicialmente em três subclasses Cl_PracticeElement, Cl_PracticesGroup e Cl_PracticesSystem (respectivamente elemento de prática, grupo de práticas e sistema de práticas) que são especializadas em outras classes [Figura 4].

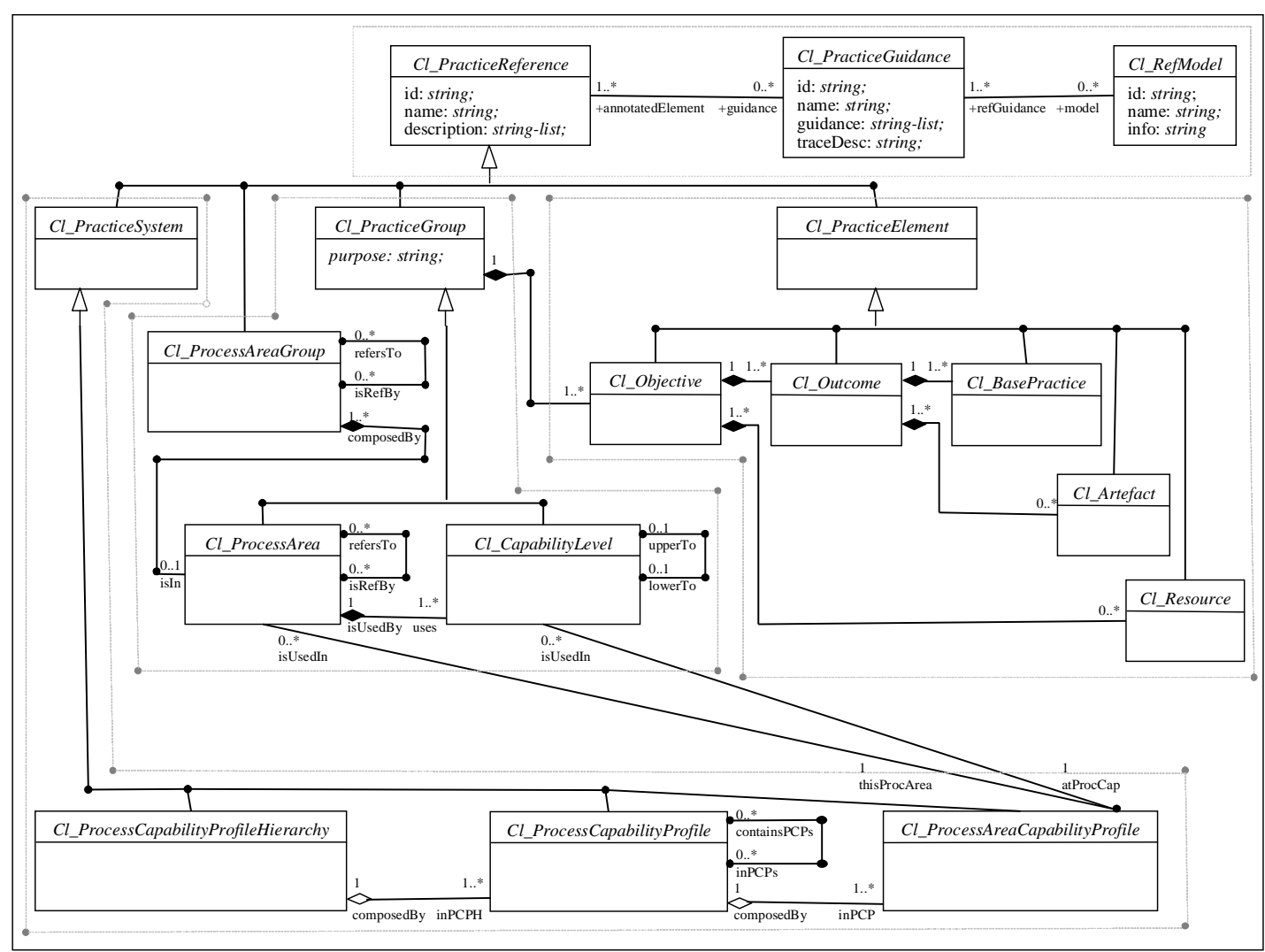

Figura 4 - Diagrama de classes do modelo de PRO2PI na notação UML 
O terceiro componente é um conjunto inicial de medições relacionadas a PRO2PI, incluindo uma medição da complexidade de um PRO2PI e da viabilidade de um PRO2PI. O quarto componente é um ciclo de melhoria com PRO2PI composto por fases correspondentes às fases dos ciclos de melhoria convencionais, principalmente, IDEAL, Ciclo 15504 e AMP1, acrescido de uma atividade para definição e utilização de PRO2PI que pode ser utilizada em qualquer fase [Figura 5].

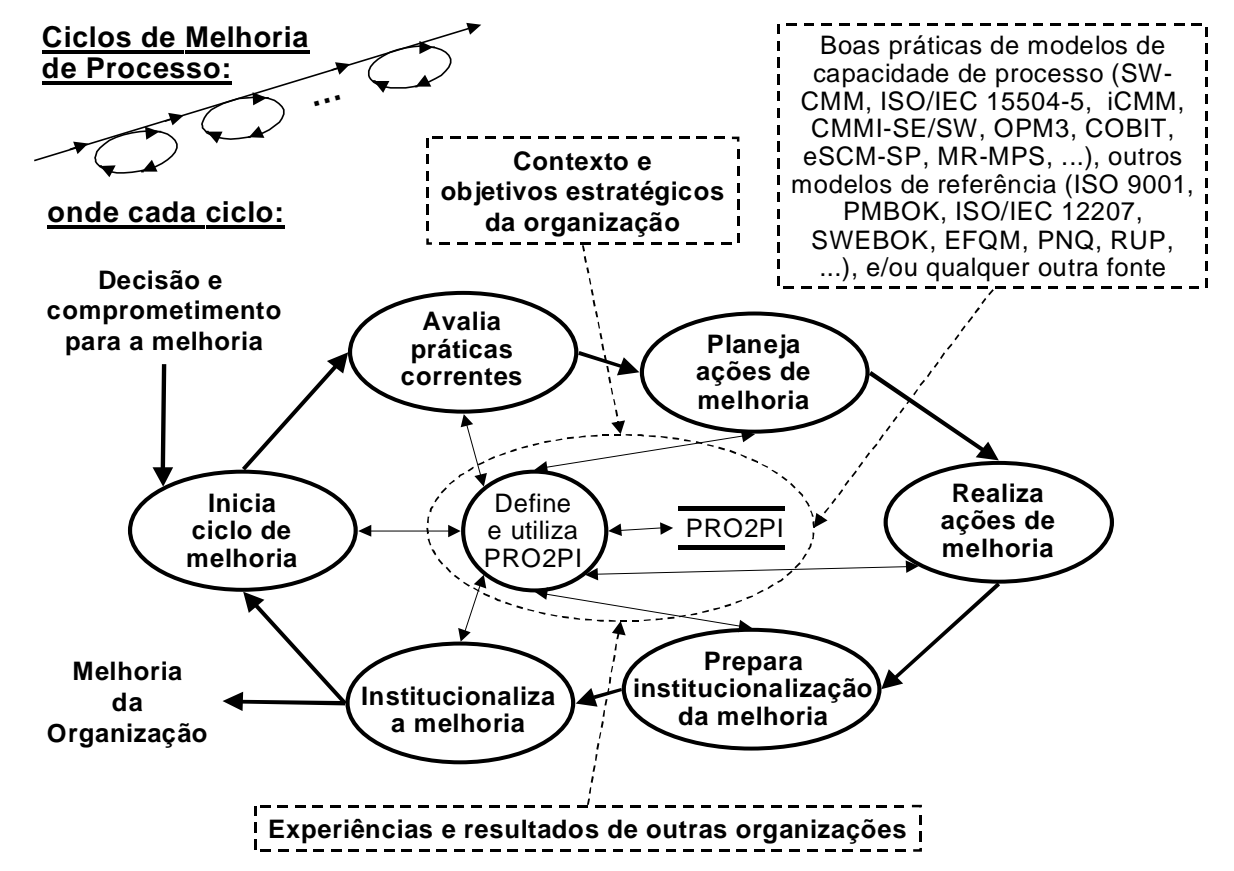

Figura 5 - Ciclo de melhoria de processo com PRO2PI

\section{Proposta 4: Método para estabelecimento de PRO2PI}

A quarta proposta é um método para estabelecimento de PRO2PI como início de um ciclo de melhoria em micro e pequenas organizações com processos de baixa capacidade que queiram iniciar um ciclo de melhoria o mais rápido possível, investindo poucos recursos e com resultados de curto prazo. Em geral o método pode ser realizado em 5 dias de trabalho, orienta melhorias que podem ser realizadas pelos próprios membros da organização, com resultados em cerca de 4 a 6 meses. A estratégia é identificar melhorias importantes para a organização, que possam ser realizadas com seus próprios recursos humanos e tenham resultados a curto prazo.

Esse método realiza de forma compactada as atividades das três primeiras etapas do ciclo de melhoria de PRO2PI mais as atividades de definição e utilização de PRO2PI, e também as quatro primeiras etapas do ciclo de melhoria da ISO/IEC $15504 \mathrm{e}$ as três primeira etapas do ciclo IDEAL [McFeeley 1996].

O propósito do método é estabelecer um perfil de capacidade de processo como indutor de um ciclo de melhoria de processo (PRO2PI). Este propósito é desdobrado nos seguintes objetivos específicos: (i) Capacitar membros da organização em fundamentos da melhoria de processo e de modelos relevantes de capacidade de processo, (ii) Consolidar informações relevantes sobre a organização, (iii) Consolidar objetivos estratégicos para a melhoria, (iv) Definir um PRO2PI, (v) Entender os processos atuais em relação ao PRO2PI definido, (vi) Consolidar uma representação em 
alto nível dos processos atuais, (vii) Definir orientações para atingir PRO2PI, e (vii) Reforçar motivação para melhoria de processo.

O método é definido em quatro fases (Preparação para o trabalho, escolha de PRO2PI, orientações para PRO2PI e conclusão do trabalho) com um total de vinte atividades. Cada fase é descrita em termos do propósito, critérios de entrada, critérios de saída e relação das atividades. Cada atividade é descrita em termos de propósito, produtos de entrada, produtos de saída, tarefas e opcionalmente, outros elementos e exemplos da atividade.

A estratégia para definição do perfil de capacidade de processo é baseada na consolidação de informações relevantes sobre a organização, análise de um conjunto de áreas de processo em relação à organização e escolha das áreas de processo. As informações sobre a organização incluem pontos fortes, fraquezas, oportunidades, ameaças e objetivos de negócio. São levantadas informações objetivas que servem de subsídios para uma decisão subjetiva e coletiva.O método deve ser realizado como um processo de no mínimo nível 3 de capacidade. Ou seja, como um processo executado, gerenciado e estabelecido. Os parâmetros para adaptação do método PRO2PI incluem características do tipo de trabalho, da distribuição dos participantes, perfil dos participantes e a abrangência. Quanto à abrangência, pode ser para uma determinada área de processo, algumas áreas de processo de um ou mais modelos, áreas de processo de um único modelo ou totalmente aberto.

Uma variação do método pode ser utilizado para ensino da melhoria de processo, com por exemplo em disciplinas relacionadas à melhoria de processo em cursos de pós-graduação (especialização) relacionados a engenharia e qualidade de software e em treinamentos para projetos de melhoria de processo. Esta utilização segue uma visão construtivista do aprendizado. Esta visão construtivista é baseada informalmente na linha de educação defendida, entre outros, por Freire [1996, p. 25]. Os participantes são geralmente profissionais em software, com necessidades de conhecimento úteis ao seu dia-a-dia e com pouco tempo para aprendizado. Com PRO2PI-WORK "ensinar não é transferir conhecimento, mas criar as possibilidades para a sua produção ou a sua construção" [Freire 1996, p. 25].

\section{Experimentações e análises}

A Tabela 1 sintetiza os tipos de utilizações, a quantidade de projetos, e a quantidade de utilizações nesses projetos, dos fundamentos e elementos de PRO2PI.

Tabela 1 - Síntese das utilizações de PRO2PI

\begin{tabular}{|l|l|l|}
\hline \multicolumn{1}{|c|}{ Característica e período da utilização } & Projetos & \multicolumn{1}{|c|}{ Usos } \\
\hline Melhoria de processo de software na Empresa E1 [1999-2002] & 1 & 3 \\
\hline Melhoria de processo de software na Empresa E2 [2002-2003] & 1 & 1 \\
\hline Estabelecimento de perfil de capacidade de processo [2000-2005] & 9 & 12 \\
\hline Projeto 15504MPE [2003-2004] & 1 & 5 \\
\hline Melhoria de processo em grupos de empresas [2004-2005] & 2 & 8 \\
\hline PRO2PI para domínios específicos [2004-2005] & 7 & 7 \\
\hline Cursos com PRO2PI-WORK [2004-2005] & 10 & 164 \\
\hline TOTAL & 32 & 200 \\
\hline
\end{tabular}


Ao todo, os fundamentos e elementos de PRO2PI foram utilizados em 32 (trinta e dois projetos) e nesses projetos houve um total de 200 (duzentas) utilizações de PRO2PI, principalmente relacionados ao estabelecimento de um perfil de capacidade de processo para orientar um ciclo de melhoria em organizações intensivas em software.

Também foram realizadas análises dos modelos de capacidade de processo mais relevantes em relação às três gerações de arquitetura propostas, a estabilidade e variabilidade do uso dos três conceitos principais (perfil de capacidade de processo, área de processo e nível de capacidade de processo) e aos elementos do modelo unificado de PRO2PI proposto.

\section{Principais contribuições da pesquisa}

As contribuições principais desta tese estão sintetizadas na Figura 6 em termos dos objetivos, propostas e validações, e os relacionamentos entre eles.

\begin{tabular}{|c|c|c|}
\hline Objetivos: & Propostas: & Validações: \\
\hline Objetivo 1: & rês geracões de arquitetura & \\
\hline $\begin{array}{l}\text { investigar arquiteturas } \\
\text { estagiada e contínua }\end{array}$ & $\begin{array}{l}\text { modelos de capacidade de } \\
\text { processo: estagiada fixa, contínua }\end{array}$ & Validacão 1: \\
\hline Objetivo 2: & Menada contrinua auerta & análise \\
\hline $\begin{array}{l}\text { evoluir melhoria de } \\
\text { processo para modelos }\end{array}$ & engenharia de processo PCDE & $\begin{array}{l}\text { em relação a } \\
\text { modelos }\end{array}$ \\
\hline contínuos & Proposta 3: & \\
\hline Objetivo 3: & abordagem PRO2PI com & \\
\hline $\begin{array}{c}\text { desenvolver } \\
\text { abordagem para esta }\end{array}$ & $\begin{array}{l}\text { propriedades, modelo, ciclo } \\
\text { de melhoria e medições }\end{array}$ & \\
\hline melhoria evoluída & osta 4: & \\
\hline Objetivo 4: & método para & \\
\hline $\begin{array}{l}\text { exercitar a abordagem } \\
\text { principalmente em micrc } \\
\text { e pequenas empresas }\end{array}$ & ecimento de D & análise das \\
\hline Objetivo 5: & Proposta 5: & \\
\hline estabelecer agenda de & agenda de pesquisa, & alidação 3: \\
\hline $\begin{array}{ll}\mathrm{A} & \\
\mathrm{X} & \mathrm{X} \\
\end{array}$ & $\begin{array}{l}\text { Ima proposta para o objetivo } \mathrm{A} \\
\text { Ima validação para a proposta } \mathrm{X}\end{array}$ & $\begin{array}{l}\text { continuação da } \\
\text { pesquisa } \\
\text { novas pesquisas } \\
\end{array}$ \\
\hline
\end{tabular}

Figura 6 - Objetivos, propostas e validações da pesquisa

A primeira contribuição (Proposta 1) é a identificação de três gerações de arquitetura de modelos de capacidade de processo, organizadas pela estabilidade e variabilidade dos conceitos de níveis de capacidade de processo, áreas de processo e perfis de capacidade de processo. Estas três gerações substituem a caracterização atual de arquitetura estagiada e contínua. Esta contribuição atende ao Objetivo 1 e é validada pela Validação 1, Validação 2 e pelo desenvolvimento da Proposta 2.

A segunda contribuição (Proposta 2) é a proposta da engenharia de processo PCDE), como evolução da atual melhoria de processo de software baseada em modelos de capacidade de processo. Esta proposta atende ao Objetivo 2 e o seu desenvolvimento é uma validação da Proposta 1. Esta proposta é validada pela Validação 2 e pelo desenvolvimento da Proposta 3. 
A terceira contribuição (Proposta 3) é o desenvolvimento e utilização da abordagem exemplo desta engenharia de processo (abordagem PRO2PI) para melhoria de processo dirigida por perfil de capacidade de processo. Esta abordagem é definida por um conjunto de propriedades, um modelo, um ciclo de melhoria e um conjunto de medições. Esta proposta atende ao Objetivo 3 e o seu desenvolvimento é uma validação da Proposta 2. Esta proposta é validada pela Validação 2.

A quarta contribuição (Proposta 4) é o desenvolvimento e utilização do método para estabelecimento de um perfil de capacidade de processo para a melhoria para capacitação em engenharia de processo e início de um ciclo de melhoria principalmente em micro e pequenas empresas. Esta proposta atende ao Objetivo 3 e o seu desenvolvimento é uma validação da Proposta 3. Esta proposta é validada pela Validação 3. A quinta contribuição (Proposta 5) é o estabelecimento de uma agenda de pesquisa e desenvolvimento em engenharia de processo dirigida por perfis de capacidade de processo. Esta proposta atende ao Objetivo 5. Esta quinta contribuição orienta a continuação dos trabalhos é validada pela Validação 4.

\section{Conclusão}

PCDE e PRO2PI são extensões do modelo conceitual definido pela ISO/IEC 15504. Os resultados dessa pesquisa podem ser relacionados e comparados com pelo menos quatro outras propostas similares. PRO2PI apoia a definição e utilização de perfis dinâmicos e representativos do processo, com elementos de qualquer fonte, o que não é suportado pelas abordagens RAPID [Rout et al. 2000], Constagedeous [Kasse 2004] e CMMI in Small Settings Toolkit [Garcia et al. 2005] e MARES [Anacleto 2005].

Quatro limitações principais foram identificadas neste trabalho, relacionadas respectivamente ao uso dos níveis de capacidade de processo, validação mais qualitativa das propostas, o uso maior de apenas cinco das oitos propriedades e as restrições do contexto de aplicação do método de estabelecimento de PRO2PI.

Um dos objetivos dessa pesquisa foi o estabelecimento de uma agenda para continuação dos trabalhos. Dezesseis ações são sugeridas, algumas já iniciadas, que envolvem, entre outras, um maior detalhamento e utilizações das propostas, exploração de uma análise semiótica de PCDE e PRO2PI, o estabelecimento de um ambiente livre para evolução e exemplos de utilização de PCDE e PRO2PI, e o desenvolvimento de hierarquia de perfis de capacidade de processo para segmentos ou domínios específicos, como, por exemplo, para o domínio de micro e pequenas empresas orientadas ao desenvolvimento, comercialização e evolução continua de uma sistema.

Ao todo, os fundamentos e elementos das propostas dessa pesquisa foram utilizados em pelo menos 200 (duzentas) aplicações diretas ou indiretas em organizações de software. A quantidade de utilizações e a receptividade de sua proposta descrita juntamente com outros trabalhos relacionados indicam a viabilidade das propostas. Alguns resultados da utilização de PRO2PI foram comparados com resultados de outras abordagens, incluindo outros trabalhos em Santa Catarina e na Finlândia [von Wangenheim et al. 2006], e foram constatadas similaridades entre os resultados principalmente na diversidade de perfis de capacidade de processo estabelecidos. As propostas foram também discutidas em duas das comunidades mais relevantes da área, no caso a comunidade do CMMI [Salviano e Jino 2004] e da 
ISO/IEC 15504 [Salviano et al. 2004]. Estas discussões e aplicações dos fundamentos e elementos das propostas, em conjunto com o desenvolvimento com a abordagem indústria-como-laboratório, provêm uma confiança adequada da qualidade de PCDE e PRO2PI como propostas para a evolução da melhoria de processo de software.

\section{Agradecimentos}

A várias pessoas, pela amizade e carinho, e entidades, incluindo o CenPRA e o CNPq pelo apoio, oportunidade, compreensão e financiamento do trabalho.

\section{Referências bibliográficas}

[Abran et al. 2004] Alain Abran and James W. Moore (executive editors) Pierre Bourque, Robert Dupuis and Leonard L. Tripp (editors), Guide to the Software Engineering Body of Knowledge - SWEBOK, 2004 version, The Institute of Electrical and Electronics Engineers, Inc. - IEEE, 210 pages, 2004.

[Anacleto 2004] Alessandra Anacleto, "Método e modelo de avaliação para melhoria de processos de software em micro e pequenas empresas", Dissertação de mestrado, Programa de pós-graduação em Ciência da Computação da Universidade Federal de Santa Catarina, 173 páginas, 2004.

[Bézivin 2003] Jean Bézivin, MDA TM : From Hype to Hope and Reality, Slides from a guess talk presentation at UML'2003, San Francisco, 96 slides, 2003.

[Brooks 1986] Frederick P. Brooks, Jr., No Silver Bullet, in Proceedings of the IFIP Tenth World Computing Conference, pp. 1069-1076, 1986.

[Card 2004] David N. Card, Research Directions in Software Process Improvement, Proceedings of COMPSAC 2004, Design and Assessment of Trustworthy Software-Based Systems, Hong Kong, China, IEEE, p. 238, 27-30 September 2004.

[Chrissis et al. 2003] Mary Beth Chrissis, Mike Konrad and Sandy Shrum, CMMI: Guidelines for Process Integration and Product Improvement, Addison-Wesley Pub Co, 2003.

[Conradi e Fuggetta 2002] Reidar Conradi and Alfonso Fuggetta, Improving Software Process Improvement, IEEE Software, 19(4), p. 92-99, July/August 2002.

[Curtis 1998] Bill Curtis, "Which Comes First, the Organization or its Processes", IEEE Software, pages 10-13, November/December 1998.

[Drucker 1992] Peter Drucker, Managing for the future: The 1990s and beyond, 1992.

[El Emam 2004] Khaled El Emam, Software Engineering Process, Chapter 9 of Guide to the Sw. Engineering Body of Knowledge (SWEBOK), 2004 version, p. 9-1 to 9-14, 2004.

[Freire 1996] Paulo Freire, Pedagogia da autonomia - Saberes necessários à prática educativa, Coleção Leitura, Editora Paz e Terra, 1996.

[Garcia et al. 2004] Suzane Garcia, Sandra Cepeda, Gene Miluk and Mary Jo Staley, Adopting CMMI for Small Organizations, slides from presentation at Fourth 
Annual CMMI Technology Conference and Users Group, Denver, USA, November 2004.

[Herbsleb et al. 1997] James Herbsleb, David Zubrow, Dennis Goldenson, Will Hayes and Mark Paulk, Software Quality and the Capability Maturity Model, in Communication of the ACM, Vol. 40, No. 6, pp. 30-40, June 1997.

[Ibrahim 2000] Linda Ibrahim, Using an Integrated Capability Maturity Model® - The FAA Experience, in Proceedings of the Tenth Annual International Symposium of the International Council on Systems Engineering (INCOSE), USA, pp. 643-648, July 2000.

[ISO/IEC 15504-2 2003] The International Organization for Standardization and the International Electrotechnical Commission, ISO/IEC 15504 - Information Technology - Process Assessment - Part 2, 2003.

[ISO/IEC 15504-5 2006] ISO/IEC 15504 - Information Technology - Process Assessment - Part 5: An exemplar Process Assessment Model, 2006.

[ISO/IEC TR 15504-5 1998] ISO/IEC TR 15504-5 - Information Technology Software Process Assessment - Part 5 : An assessment model and indicator guidance, Technical Report, 1998.

[Kasse 2004] Tim Kasse, Practical Insight into CMMI, Artech House Publishers, 2004.

[McFeeley 1996] Bob McFeeley, IDEAL - A User's Guide for Software process Improvement, Handbook CMU/SEI-96-HB-001, 236 pages, 1996.

[Naur e Randell 1969] Peter Naur and Brian Randell (Editors), Software Engineering: Report on a conference sponsored by the NATO Science Committee, Garmisch, Germany, 7-11 October 1968, 136 pages, January 1969.

[Olson 2003] Timothy G. Olson, Staged or Continuous: Which Model Should I Choose?, slides from presentation at Third Annual CMMI Technology Conference and Users Group, USA, November 2003.

[Paulk et al. 1994] Mark C. Paulk, Charles V. Weber, Bill Curtis and Mary Beth Chrissis, The Capability Maturity Model - Guidelines for Improving the Software Process, CMU-SEI, Addison-Wesley, 441 pages, 1994.

[Pollice 2005] Gary Pollice, Teaching software development vs. software engineering, in The Rational Edge, 5 pages, December 2005.

[Potts 1993] Colin Potts, "Software-Engineering Research Revised", IEEE Software, Volume 10, Number 5, pages 19-28, September 1998.

[Rifkin 2002] Stan Rifkin, Is process improvement irrelevant to produce new era software? in Software Quality - ECSQ 2002, Lecture Notes in Computer Science 2349, ed. by Jyrki Kontio and Reidar Conradi, pp. 13-16, Springer-Verlag, 2002.

[Rout et al. 2000] Terry Rout, Angela Tuffley, Brent Cahill and B. Hodgen, The Rapid Assessment of Software Process Capability, Proceedings of SPICE 2000 The First International SPICE Conference, June 10-11, 2000, Limerick, Ireland, p. 47-55. 
[Salviano 2006] Clênio F. Salviano, Uma proposta orientada a perfis de capacidade de processo para evolução da melhoria de processo de software, Tese de doutorado, Faculdade de Engenharia Elétrica e de Computação da Universidade Estadual de Campinas (FEEC-Unicamp), 2006.

[Salviano e Jino 2004] Clênio F. Salviano and Mario Jino, Using Continuous Modes as "Dynamic and Specific Staged Models", slides from presentation at Fourth Annual CMMI Technology Conference and Users Group, Denver, USA, November 2004.

[Salviano et al. 2004] Clênio F. Salviano, Mario Jino and Manuel de Jesus Mendes, Towards an ISO/IEC 15504-Based Process Capability Profile Methodology for Process Improvement (PRO2PI), in Proceedings of SPICE 2004 The Fourth International SPICE Conference, Lisbon, Portugal, p. 77-84, April 28-29, 2004.

[Shaeffer 2004] Mark Shaeffer, DoD System Engineering and CMMI, slides from keynote speaker at Fourth Annual CMMI Technology Conference and Users Group, Denver, USA, November 2004.

[Shaw 1990] Mary Shaw, Prospects for an Engineering Discipline of Software, IEEE Software, November 1990.

[von Wangenheim et al. 2006] Christiane G. von Wangenheim, Timo Varkoi and Clênio F. Salviano, Process Assessments in Small Software Companies, to be published at SPIP Software Process Improvement and Practice Journal, 2006.

[Weber et al. 2005] Kival C. Weber, Eratóstenes Araújo, Cristina A. F. Machado, Danilo Scalet, Clênio F. Salviano, e Ana Regina C. da Rocha, Modelo de Referência e Método de Avaliação para Melhoria de Processo de Software versão 1.0 (MR-MPS e MA-MPS), Anais do IV Simpósio Brasileiro de Qualidade de Software, SBQS, p. 347-360, Porto Alegre, RS, 6 a 10 de junho de 2005.

[Zahran 1998] Sami Zahran, Software Process Improvement - Practical Guidelines for Business Success, Addison-Wesley, 447 pages, 1998. 